\title{
The learning curve of real time elastosonography: a preliminary study conducted for the assessment of malignancy risk in thyroid nodules
}

\author{
Idil Gunes Tatar' ${ }^{1}$ Aydin Kurt' ${ }^{1}$ Kerim Bora Yilmaz², Melih Akinci², Hakan Kulacoglu², \\ Baki Hekimoglu' ${ }^{1}$
}

${ }^{1}$ Department of Radiology, Diskapı Training and Research Hospital, ${ }^{2}$ Department of General Surgery, Diskapi Training and Research Hospital, Ankara, Turkey

\begin{abstract}
Background: Malignancy is correlated with stiffness which is assessed by real time elastosonography (RTE). RTE is not used in routine practice. We aimed to establish the learning curve of RTE on radiology residents. Methods: Forty $\geq 1 \mathrm{~cm}$ solitary thyroid nodules referred to fine needle aspiration cytology were examined with RTE by a radiology specialist and two radiology residents separately. Patients with malignant and undetermined FNAC findings underwent surgery. Strain ratio and elasticity score results of the radiology residents were compared to the results of the radiology specialist taking the histopathology results as the reference. To establish the learning curve and compare the diagnostic accuracy of residents, Receiver Operating Characteric curves were generated and Area Under the Curve were calculated. Results: Thirty-four nodules were benign and the others were malignant. The radiology specialist had a very high correct class prediction for the differentiation of benign versus malignant thyroid nodules. Statistical analysis of the strain ratio measurements showed that one of the residents had similar results with the radiology specialist after the seventh patient and the other one after the fourth patient. On the other hand the elasticity score measurements of all examiners had low correct class prediction. Conclusions: Strain ratio measurement by RTE is an easily learned sonographic method that can assist in the evaluation of benign versus malignant nature of the lesions. However, interpretation of the elasticity scores requires more expertise. The results of this preliminary study need to be verified with a larger sample population.
\end{abstract}

Keywords: thyroid, elastosonography, nodule, learning curve

\section{Introduction}

Thyroid nodules are detected by palpation in 3-7\% and by B-mode sonography (US) in $20-76 \%$ of the general population [1]. The clinical importance of thyroid nodules is the possibility of cancer, which can occur in 5\% of thyroid nodules [2]. Fine Needle Aspiration Cytology

Received 27.08.2013 Accepted 15.09.2013

Med Ultrason

2013, Vol. 15, No 4, 278-284

Corresponding author: Idil Gunes Tatar, M.D.

Department of Radiology,

Ankara Diskapi Training and Research

Hospital, 06110,

Altındag/ Ankara - TURKEY

Phone:+90312 5962616

E-mail: idilttr@yahoo.com
(FNAC) is the established method to distinguish between benign versus malignant nodules. US acts as the preselection modality for FNAC [3]. Some sonographic criteria may increase suspicion for malignancy such as hypoechoic texture, ill-defined contours, presence of microcalcifications, antero-posterior diameter greater than transverse diameter [4]. The diagnostic value of the suspicious US features have been reported to have low sensitivity [5]. Real Time Elastosonography (RTE) has recently emerged as a sonographic method which measures the tissue stiffness and aids in the differentiation of the benign versus malignant nature [6]. The use of RTE has still not been established in routine practice. Many residents and specialists who are not familiar with the technique do not utilize RTE even if they have the elastography application on their device. The user dependant nature of the technique, 
hence the lack of experience, may cause drawbacks. The required experience for competency is unknown.

RTE can potentially aid in the management of thyroid lesions [7]. Thyroid cancer generally occurs as a stiff lesion and RTE has been used in predicting thyroid cancer $[8,9]$. According to the Revised American Thyroid Association Management Guidelines, elastography is an emerging and promising sonographic technique that requires additional validation with prospective studies [10].

The aim of this study is to establish the learning curve of RTE technique on thyroid nodules for radiology residents.

\section{Materials and methods}

A total of 40 consecutive patients ( 30 females, 10 males) with sonographically diagnosed solitary thyroid nodules between November 2012 and June 2013, were considered for this prospective study. The inclusion criterion was the presence of a single thyroid nodule larger than $1 \mathrm{~cm}$. The exclusion criteria were nodules with $>50 \%$ cystic portion and multinodular goiter. The patient flow of the study is summarized in figure 1 . This study was approved by the institutional review board and informed consent was obtained from all patients. US and RTE examinations were performed by a radiology specialist with twelve years of sonography experience and two senior radiology residents separately. The examiners were independent and did not take part in the preparation of the manuscript.

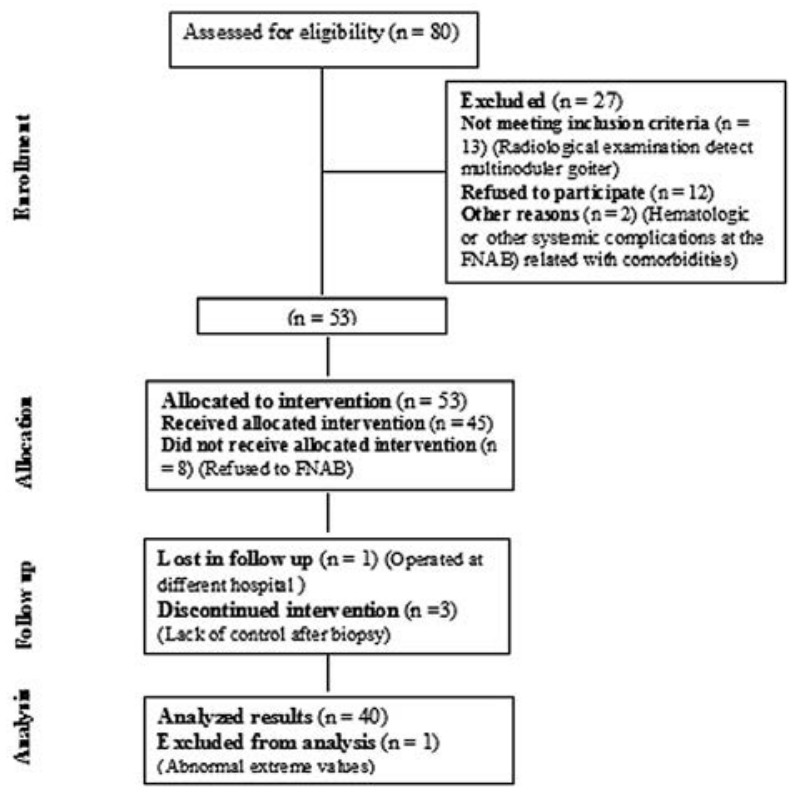

Fig 1. Outline of study design followed by the Consolidated Standards of Reporting Trials (CONSORT) statement.
Conventional B-mode US and RTE imaging

For the examination of the thyroid nodules, $4-13 \mathrm{MHz}$ linear probe of Ultrasonography (ESAOTE Gold Platform MayLab 60, Italy) equipped with elasto software (elaxto) which was not modified was used. First B-mode images were obtained for each nodule (fig 2). Afterwards RTE was performed by applying light vertical pressure followed by decompression until a good quality image was obtained. US and RTE images were demonstrated next to each other on the screen.

A region-of-interest (ROI) box was placed covering the majority of the nodule (average strain represented as A) taking the adjacent normal thyroid tissue at the same depth (average strain represented as B) as the reference. Strain ratio (B/A) which reflects the stiffness of the lesion was calculated for each nodule separately by three examiners blinded to each other (fig 3).

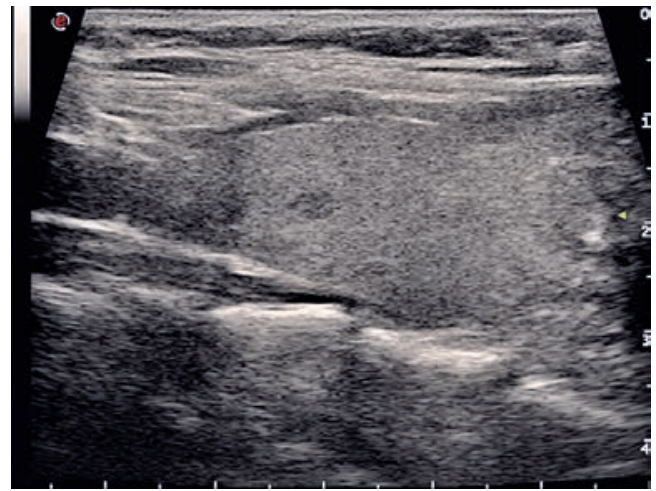

Fig 2. B-mode sonographic examination by the radiology specialist revealed a solitary hypoechoic solid nodule in the right lobe of thyroid gland in a 38 year-old female patient.

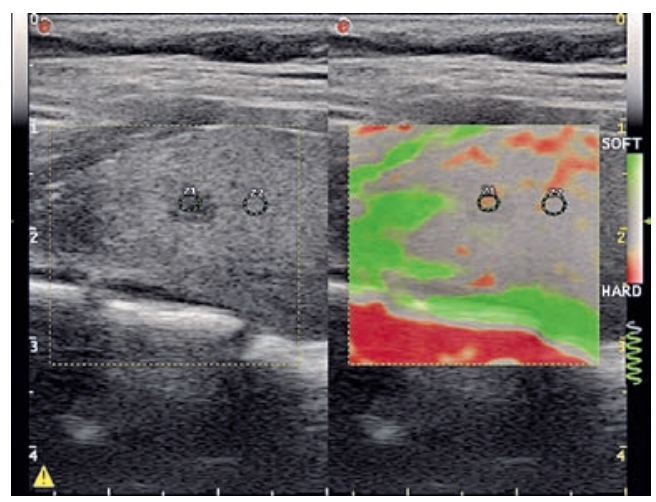

Fig 3. Strain ratio $(B / A)$ was measured 1.31 by the radiology specialist with real time elastosonography (A: average strain of the nodule, B: average strain of the thyroid gland (same patient). 


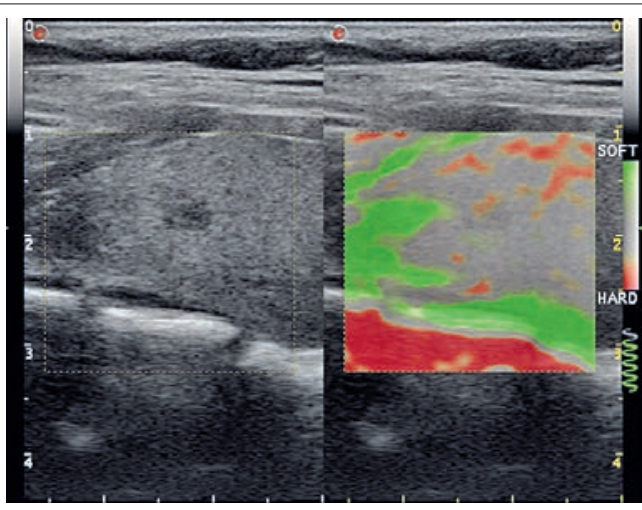

Fig 4. Elasticity score of the nodule was interpreted as 2 (mainly elastic) by the radiology specialist (same patient).

The elastogram based on a color scale was displayed on the B-mode image which ranges from blue to red. Blue represented tissue with greatest elastic strain meaning softest components, while red represented tissue with no strain meaning hardest components. The elasticity scores of the nodules were classified into 4 types as proposed by Rubaltelli et al. and were separately interpreted by three examiners blinded to each other [11] (Table I, fig 4). Scores 3 and 4 were assumed to be indicative of malignancy.

FNAC

All patients underwent US guided FNAC by another radiology specialist later on the same day. Cytology results were taken as the reference standard for the benign nodules. Patients with malignant and undetermined FNAC findings underwent surgery and histopathology results were taken as the reference standard.

\section{Statistical Analysis}

All statistical analyses were performed using SPSS version 18.0 (SPSS Inc, Chicago, IL, USA). Strain ratio and elasticity score results obtained by the radiology residents were compared to the results of the radiology specialist taking the histopathology results as the reference. To establish the learning curve and compare the diagnostic accuracy of residents, ROC (Receiver Operating Characteric) curves were generated and AUC (Area Un-

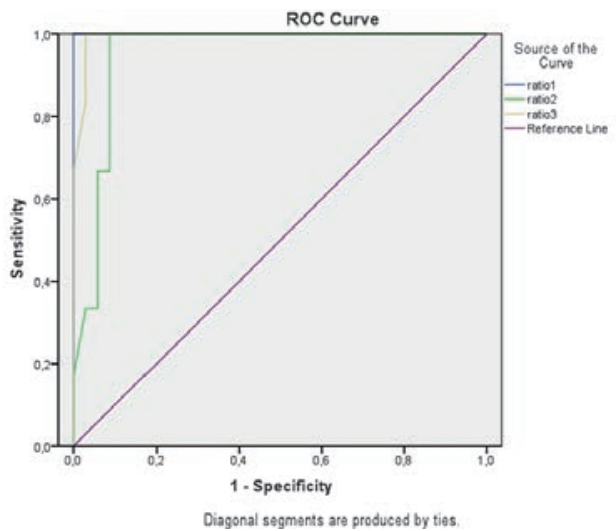

Fig 5. Receiver Operating Characteric curve analysis of the strain ratio measurements of the three examiners (ratio 1: radiology specialist, ratio 2: first resident, ratio 3: second resident).

der the Curve) were calculated. ROC curve was formed by plotting the relationship of the true positivity (sensitivity) and the false positivity ( 1 - specificity) at various cut-off points of the tests. An AUC of 1.0 is characteristic of an ideal test, whereas 0.5 indicates a test of no diagnostic value [12].

\section{Results}

The mean age of the patients was $42.95 \pm 9.98$ years (age range, 24-71years). The mean anteroposterior diameter was $17.80 \pm 4.49 \mathrm{~mm}$ (range, 11-35) and transverse diameter was $16.62 \pm 3.76 \mathrm{~mm}$ (range,12-29). Thirty-four thyroid nodules had benign cytology results. Four nodules had malignant cytology results and their histopathology proved to be papillary carcinoma. Two nodules with undetermined FNAC findings were histopathologically proved to be papillary carcinoma and follicular carcinoma.

ROC curve analysis of the strain ratio measurements by the three examiners was carried out (fig 5, table II). The radiology specialist had very high correct class prediction for the differentiation of benign versus malignant thyroid nodules $(\mathrm{AUC}=1.0, \mathrm{p}=0.000$ ). The first resident started to have similar results with the radiology specialist after the seventh patient (AUC $=0.993, \mathrm{p}=0.000$ ). The

Table I. Elasticity Scores

\begin{tabular}{ll}
\hline 1 & Presence of elasticity in the entire nodule \\
2 & Mainly elastic nodule with presence of inelastic areas not constant during the course of the real time examination \\
3 & Presence of ample and constant inelastic areas prevalently arranged at the periphery or at the center of the nodule \\
4 & Completely inelastic nodule
\end{tabular}


Med Ultrason 2013; 15(4): 278-284

Table II. Analysis of the area under the curves for strain ratio measurements

Area Under the Curve

\begin{tabular}{|c|c|c|c|c|c|}
\hline \multirow{2}{*}{ Test Result Variable(s) } & \multirow{2}{*}{ Area } & \multirow{2}{*}{ Standard Error } & \multirow{2}{*}{ Asymptotic Significance } & \multicolumn{2}{|c|}{ Asymptotic 95\% CI } \\
\hline & & & & Lower Bound & Upper Bound \\
\hline Radiology specialist & 1.000 & .000 & .000 & 1.000 & 1.000 \\
\hline First resident & .949 & .034 & .001 & .000 & 1.000 \\
\hline Second resident & .993 & .010 & .000 & .000 & 1.000 \\
\hline
\end{tabular}

CI - Confidence Interval

Table III. Analysis of the area under the curves for elasticity scores

\begin{tabular}{lccccc}
\hline & \multicolumn{3}{c}{ Area Under the Curve } \\
\hline Test Result Variable(s) & Area & Standard Error & Asymptotic Significance & \multicolumn{2}{c}{$\begin{array}{c}\text { Asymptotic 95\% CI } \\
\text { Lower Bound }\end{array}$} \\
& & & .004 & .689 & 1.000 \\
Radiology specialist & .873 & .094 & .028 & .588 & .981 \\
First resident & .784 & .100 & .006 & .672 & 1.000 \\
Second resident & .858 & .095 & & \\
\hline
\end{tabular}

CI - Confidence Interval

second resident had similar results with the radiology specialist after the fourth patient $(\mathrm{AUC}=0.949, \mathrm{p}=0.001$ ). Cut-off point was set as 3.25 for the differentiation of benign versus malignant nodules by taking the strain ratio measurements of the radiology specialist into consideration.

According to the ROC curve analysis of the elasticity score measurements all three examiners had low correct class prediction (table III). The radiology specialist had the best results among the three examiners $(\mathrm{AUC}=0.873$, $\mathrm{p}=0.004)$. The first resident $(\mathrm{AUC}=0.858, \mathrm{p}=0.006)$ and the second resident $(\mathrm{AUC}=0.784, \mathrm{p}=0.028)$ displayed similar results to the specialist after the seventh patient.

\section{Discussion}

Radiology is a continuously developing specialty of medicine which is highly affected by the technological innovations. The core residency programme covers main aspects for all modalities and organ systems and should be based on the learning curves of the techniques. Hence, the learning curve of radiology techniques during medical education, radiology residency and practice have gained importance in the medical literature in the recent years.

Jang et al conducted a study to evaluate the learning curve of right upper quadrant (RUQ) emergency department bedside ultrasonography (EUS) for emergency physicians in training. In this study 1837 patients with suspected biliary tract disease underwent RUQ EUS by 127 physicians followed by abdominal ultrasonography (AUS) by the department of radiology for comparison. Until 50 examinations there was no significant improvement in the sensitivity or specificity for every increase in 10 examinations. For every 10 additional examinations, no improvement was observed in the detection of the sonographic findings except for pericholecystic fluid [13].

Jang et al carried out another study to assess the learning curve of emergency department bedside sonography (EBS) of emergency physicians in training for first-trimester pregnancy complications. They enrolled 670 patients with first-trimester vaginal bleeding or pain that underwent EBS by 1 of 25 physicians who would perform at least 25 examinations followed by pelvic sonography by the radiology department. Authors concluded that a satisfactory learning curve would persist after 40 examinations [14].

Boursier et al compared the liver stiffness examination (LSE) of 5 novice observers of different medical status with those of five expert observers (physicians with $>100$ examinations) in 250 patients with chronic liver disease to evaluate a LSE learning curve. They declared that LSE does not require a learning curve; a novice is able to obtain a reliable result after a single training session although the success rate will progressively increase [15]. 
In our study one of the residents started to have similar SR measurements with the radiology specialist after the seventh patient and the other after the fourth patient. Our SR measurement results are in accordance with the elastography study of Boursier et al since both studies concluded that the tecnique can be easily learned. On the other hand Jang et al declared that more practise is necessary to be able to accurately perform bedside ultrasonography for right upper quadrant and for first-trimester pregnancy complications. The difference in the results of these studies could be explained by the more user dependant and subjective nature of the sonographic interpretation. The studies of Jang et al were also conducted in the emergency department and the learning curves were not established on radiology residents. In our study the low class prediction of elasticity scores can also be attributed to the subjective nature of interpretation.

RTE is a new technique which has not been a part of routine clinical practice. In the last decade there has been an increasing use of RTE in the evaluation of thyroid nodules since thyroid cancer generally occurs as a stiff lesion. The stiffness of lesions are dependant on the cellularity and can be detected by RTE which was first proposed by Ophir et al [16]. In a recent study by Hong et al with 145 thyroid nodules referred for surgery, a sensitivity of $88 \%$ and specificity of $90 \%$ were observed [8]. In a RTE analysis of 92 thyroid nodules, Rago et al declared the sensitivity and specificity as high as $97 \%$ and $100 \%$ in detecting malignant nodules, respectively [9]. In another study in which 86 thyroid nodules were evaluated, Asteria et al demonstrated $94.1 \%$ sensitivity and $81 \%$ specificity of RTE for the diagnosis of thyroid cancer [17].

Lyshchik et al calculated the strain ratio and set 4 as the best cut off value with $96 \%$ specificity and $82 \%$ sensitivity in a study with 52 thyroid nodules [7].

Two main sources of variability in RTE is in data acquisition and in scoring [18]. A study evaluating interobserver agreement both in data acquisition and scoring for thyroid RTE was carried out by Park et al [19]. Three radiologists independently obtained RTE data and scoring interpretation on 52 surgically resected thyroid nodules. By using external compression elastography, no interobserver agreement was detected among three radiologists. They attributed this lack of interobserver agreement mostly to the fact that the extent of compression influences the image and consequently the elasticity score. They demonstrated that variability in obtaining data is larger than that in scoring.

The sources of variability in RTE using external compression can occur during the selection of an imaging plane and applying different compression levels which creates different color patterns. Eventually the subjective nature of assigning an elasticity score based on the color pattern can contribute to the variability. All of these steps can explain the low correct class prediction demonstrated in the elasticity scores of the three examiners in our research.

On the other hand we obtained reproducible strain ratio results. In a meta-analysis of eight RTE studies including 639 thyroid nodules Bojunga et al calculated a mean sensitivity of $92 \%$ and specificity of $90 \%$ for the discrimination of malignancy by RTE. Authors indicated that RTE could be used in combination to FNAC or alone instead of FNAC in the selection of nodules for surgery [20]. Merino conducted a study in which 103 consecutive patients with 106 thyroid nodules were examined. Authors claimed that the interobserver agreement for RTE was excellent, no false-negatives were found and a negative predictive value of $100 \%$ was observed [21]. Azizi et al have recently published that the positive predictive value of ES evaluation by RTE was $36.1 \%$, equal to or greater than that of gray-scale US features. The negative predictive value was $97.2 \%$, higher than all the other predictors of malignancy in a study carried out on 912 thyroid nodules [22]. In another recent study conducted by Mehrotra et al 147 thyroid nodules were evaluated by USE prior to FNAC. They concluded that nodules which are soft on USE are very likely to be benign, hence FNAC is not neccessary. However, nodules which are intermediate or hard on USE were suggested to be evaluated by further by FNAC [23]. In this sense the accurate use of RTE can potentially reduce the number of FNACs and decrease the work load and the ecenomic burden of unnecessary FNAC on the healthcare system.

The results of the study show that radiology residents in training are capable of rapidly learning SR measurement by RTE proposing that it should also be learned rapidly by the practising radiologists. On the other hand interpretation of the elasticity scores requires more expertise since it is more subjective. RTE should be included in the core radiology residency programme and continuing education to improve the healthcare through lifelong learning. European Federation of Societies for Ultrasound in Medicine and Biology guidelines should be followed in clinical practice [24]. A larger cohort will be the subject of future work to investigate the validity of the findings.

\section{Limitations of the study}

This was a preliminary study with a limited sample size. The results of this study need to be verified with a larger sample population. The prevelance of malignancy was $15 \%$ which was high compared to the literature 
probably because the study was conducted in a tertiary hospital with dedicated oncology and surgery departments. Some of the patients involved in this study had thyroid nodules which were sonographically diagnosed elsewhere and admitted to our hospital for further work up.

\section{Conclusions}

Radiological evaluation of lesions for the differentiation of benign versus malignant nature is important because unnecessary surgical procedures may lead to complications. The radiological techniques must be taught during the core education programmes of radiology residency.

In this study conducted on thyroid nodules we conclude that elasticity score interpretations were not reproducible and had low correct class prediction due to a number of sources of variability. On the other hand strain ratio measurements had very high correct class prediction for the radiology specialist and were reproducible after the seventh patient at the latest by the radiology residents.

SR measurement by RTE is an easily learned and reproducible method yet it must be practiced under the radiology specialists' surveillence since inaccurate examinations are possible in first trials. Additonal RTE training during core radiology residency programme may help improve the radiologists' accuracy. Correlation with the histopathological results must also be a part of the learning period.

Acknowledgements: We would like to thank to Dr. Erdal Cosgun from the Hacettepe University Department of Biostatistics for his support in the statistical analysis of the study.

\section{Conflicts of interest: None}

\section{References}

1. Hegedus L. Clinical practice. The thyroid nodule. N Engl J Med 2004; 351: 1764-1771.

2. Ezzat S, Sarti DA, Cain DR, Braunstein GD. Thyroid incidentalomas. Prevalence by palpation and ultrasonography. Arch Intern Med 1994; 154: 1838-1840.

3. Gharib H, Papini E, Paschke R, et al. American Association of Clinical Endocrinologists, Associazione Medici Endocrinologi, and European Thyroid Association medical guidelines for clinical practice for the diagnosis and management of thyroid nodules: executive summary of recommendations. J Endocrinol Invest 2010; 33: 51-56.
4. Kim EK, Park CS, ChungWY, et al. New sonographic criteria for recommending fine-needle aspiration biopsy of nonpalpable solid nodules of the thyroid. AJR Am J Roentgenol 2002; 178: 687-691.

5. Frates MC, Benson CB, Charboneau JW, et al; Society of Radiologists in Ultrasound. Management of thyroid nodules detected at US: Society of Radiologists in Ultrasound consensus conference statement. Radiology 2005; 237: 794-800

6. Garra BS. Elastography: current status, future prospects, and making it work for you. Ultrasound Q 2011; 27: 177186.

7. Lyshchik A, Higashi T, Asato R, et al. Thyroid gland tumor diagnosis at US elastography. Radiology 2005; 237: 202211.

8. Hong Y, Liu X, Li Z, Zhang X, Chen M, Luo Z. Real-time ultrasound elastography in the differential diagnosis of benign and malignant thyroid nodules. J Ultrasound Med 2009; 28: 861-867.

9. Rago T, Santini F, Scutari M, Pinchera A, Vitti P. Elastography: new developments in ultrasound for predicting malignancy in thyroid nodules. J Clin Endocrinol Metab 2007; 92: 2917-2922.

10. American Thyroid Association (ATA) Guidelines Taskforce on Thyroid Nodules and Differentiated Thyroid Cancer, Cooper DS, Doherty GM, Haugen BR, et al. Revised American Thyroid Association management guidelines for patients with thyroid nodules and differentiated thyroid cancer. Thyroid 2009; 19: 1167-1214.

11. Rubaltelli L, Corradin S, Dorigo A, et al. Differential diagnosis of benign and malignant thyroid nodules at elastosonography. Ultraschall Med 2009; 30: 175-179.

12. Huguet J, Castineiras MJ, Fuentes-Arderiu X. Diagnostic accuracy evaluation using ROC curve analysis. Scand J Clin Lab Invest 1993; 53: 693-699.

13. Jang TB, Ruggeri W, Dyne P, Kaji AH. The learning curve of resident physicians using emergency ultrasonography for cholelithiasis and cholecystitis. Acad Emerg Med 2010; 17: 1247-1252.

14. Jang TB, Ruggeri W, Dyne P, Kaji AH. Learning curve of emergency physicians using emergency bedside sonography for symptomatic first-trimester pregnancy. J Ultrasound Med 2010; 29: 1423-1428.

15. Boursier J, Konate A, Guilluy M, et al. Learning curve and interobserver reproducibility evaluation of liver stiffness measurement by transient elastography. Eur J Gastroenterol Hepatol 2008; 20: 693-701.

16. Ophir J, Cespedes I, Ponnekanti H, Yazdi Y, Li X. Elastography: a quantitative method for imaging the elasticity of biological tissues. Ultrason Imaging 1991; 13: 111-134.

17. Asteria C, Giovanardi A, Pizzocaro A, et al. US-elastography in the differential diagnosis of benign and malignant thyroid nodules. Thyroid 2008; 18: 523-531.

18. Yoon JH, Kim MH, Kim EK, Moon HJ, Kwak JY, Kim MJ. Interobserver variability of ultrasound elastography: how it affects the diagnosis of breast lesions. AJR Am J Roentgenol 2011; 196: 730-736. 
19. Park SH, Kim SJ, Kim EK, Kim EJ, Son EJ, Kwak JY. Interobserver agreement in assessing the sonographic and elastographic features of malignant thyroid nodules. AJR Am J Roentgenol 2009; 193: 416-423.

20. Bojunga J, Herrmann E, Meyer G, Weber S, Zeuzem S, Friedrich-Rust M. Real-time elastography for the differentiation of benign and malignant thyroid nodules: a metaanalysis. Thyroid 2010; 20: 1145-1150.

21. Merino S, Arrazola J, Cardenas A, et al. Utility and interobserver agreement of ultrasound elastography in the detection of malignant thyroid nodules in clinical care. AJNR Am J Neuroradiol 2011; 32: 2142-2148.
22. Azizi G, Keller J, Lewis M, Puett D, Rivenbark K, Malchoff $\mathrm{C}$. Performance of elastography for the evaluation of thyroid nodules: a prospective study. Thyroid 2013; 23 : 734-740.

23. Mehrotra P, McQueen A, Kolla S, Johnson SJ, Richardson DL. Does elastography reduce the need for thyroid FNAs? Clin Endocrinol 2013; 78: 942-949.

24. Cosgrove D, Piscaglia F, Bamber J, et al. EFSUMB guidelines and recommendations on the clinical use of ultrasound elastography. Part 2: Clinical applications. Ultraschall Med 2013; 34: 238-253. 